\title{
A systematic approach for identifying and presenting mechanistic evidence in human health assessments
}

\author{
Mary E. Kushman ${ }^{1}$, Andrew D. Kraft ${ }^{2}$, Kathryn Z. Guyton², Weihsueh A. Chiư ${ }^{2}$, Susan L. \\ Makris $^{2}$, and Ivan Rusyn \\ ${ }^{1}$ Department of Environmental Sciences and Engineering, Gillings School of Global Public Health, \\ University of North Carolina at Chapel Hill, Chapel Hill, NC \\ ${ }^{2}$ National Center for Environmental Assessment, Office of Research and Development, United \\ States Environmental Protection Agency, Washington, DC
}

\begin{abstract}
Clear documentation of literature search and presentation methodologies can improve transparency in chemical hazard assessments. We sought to improve clarity for the scientific support for cancer mechanisms of action using a systematic approach to literature retrieval, selection, and presentation of studies. The general question was "What are the mechanisms by which a chemical may cause carcinogenicity in the target tissue?" Di(2-ethylhexyl)phthalate was used as a case study chemical with a complex database of $>3,000$ publications. Relevant mechanistic events were identified from published reviews. The PubMed search strategy included relevant synonyms and wildcards for DEHP and its metabolites, mechanistic events, and species of interest. Tiered exclusion/inclusion criteria for study pertinence were defined, and applied to the retrieved literature. Manual curation was conducted for mechanistic events with large literature databases. Literature trees documented identification and selection of the literature evidence. The selected studies were summarized in evidence tables accompanied by succinct narratives. Primary publications were deposited into the Health and Environmental Research Online (http:// hero.epa.gov/) database and identified by pertinence criteria and key terms to permit organized retrieval. This approach contributes to human health assessment by effectively managing a large volume of literature, improving transparency, and facilitating subsequent synthesis of information across studies.
\end{abstract}

\section{Keywords}

systematic review; mechanistic evidence; di(2-ethylhexyl)phthalate; hazard assessment

() 2013 Elsevier Inc. All rights reserved.

Corresponding author: Ivan Rusyn, MD, PhD, Department of Environmental Sciences and Engineering, University of North Carolina, Chapel Hill, NC 27599; iir@ unc.edu; Phone: 919-843-2596; Fax: 919-843-2596.

Publisher's Disclaimer: This is a PDF file of an unedited manuscript that has been accepted for publication. As a service to our customers we are providing this early version of the manuscript. The manuscript will undergo copyediting, typesetting, and review of the resulting proof before it is published in its final citable form. Please note that during the production process errors may be discovered which could affect the content, and all legal disclaimers that apply to the journal pertain.

Disclaimer: The views expressed are those of the authors and do not necessarily reflect the views or policies of the United States Environmental Protection Agency 


\section{Introduction}

Toxicological reviews conducted in support of US Environmental Protection Agency's Integrated Risk Information System (IRIS) summarize information about potential adverse health effects from long-term chemical exposures, and provide a scientific basis for Agency's decisions supporting risk management in the protection of public health. IRIS toxicological reviews address both cancer and noncancer hazards of environmental contaminants and include descriptive and quantitative information about known or possible adverse health effects. The critical examination of a large body of evidence is necessary to understand health outcomes from chemical exposures, underscoring the importance of the quality and transparency of the systematic literature review and evidence presentation. Indeed, as has been pointed out by several National Research Council (NRC) committees (NRC, 2009; NRC, 2010; NRC, 2011), comprehensive and logically-organized evidence presentation is essential for clearly describing the scientific evidence in support of conclusions about chemical hazard(s) and the related mechanisms of action.

The challenge of characterizing the evidential support for decision-making has been best addressed in the field of evidence-based medicine wherein a systematic approach to determining the quality of evidence and the strength of a particular recommendation has been adopted (Drummond et al., 2008; Guyatt et al., 2012b). Approaches such as GRADE (Grading of Recommendation Assessment, Development and Evaluation) require clear specification of a study question, and outline suggestions for tabular presentation of evidence (Guyatt et al., 2012a; Guyatt et al., 2011). Other methodologies include the Institute of Medicine approach (IOM, 2008) of an analysis framework for evaluating exposures incurred during military service and the contribution to disease in armed forces personnel. Approaches for causal inference, hierarchies for classifying strength of evidence, and for reporting meta-analyses are also hallmarks of reports that guide public health decision-making (DHHS, 2004; Stroup et al., 2000). The CAMARADES (Collaborative Approach to Meta-Analysis and Review of Animal Data from Experimental Studies) initiative, for example, seeks to address sources of bias affecting the validity of animal studies and hence translation of the results to the clinic (e.g., of candidate drugs) (van der Worp et al., 2010). However, the evidence base for supporting medical decisions, including clinical trials of intentional exposures to potential therapeutics, differs greatly from that typically used in assessing hazards of environmental or occupational exposures to chemicals. An adaption of the evidence-based medicine approach for environmental health has been proposed, focusing on the following critical aspects: 1) clearly defining the study question, and 2) documenting the evidence collection and decision-making processes, including the criteria and rationale for each choice (Woodruff and Sutton, 2011). Together, these serve as a guide for human health assessments of chemicals, with particular regard to transparency and documentation of evidence selection.

The NRC recommends several strategies for enhancement of clarity and transparency in evidence evaluation for environmental chemicals (NRC, 2009; NRC, 2010; NRC, 2011). For extensive databases, tabular presentation of the evidence is recommended to clarify what data are available for the chemical and its metabolites, including mechanistic events, species of interest, dose or concentration spacing, replicates and variability, and experimental details and results. Graphical tools, such as flow diagrams, further aid documentation of evidence capture, selection, and evaluation. Finally, public access to the systematically organized literature used to draw conclusions is needed.

In accord with the NRC guidance for improving evaluation for environmental chemicals, we aimed to devise an approach that can be applied to a systematic search, identification, and presentation of the mechanistic evidence on chemical hazards, and that would provide 
succinct and informative tabular presentation of key studies and their findings in support of human health assessment decisions. We explored as a case example the mechanistic events potentially involved in liver carcinogenesis by di(2-ethylhexyl)phthalate (DEHP), a chemical with a voluminous and complex database of mechanistic studies. The multi-step strategy applied herein was designed to address this particular topic and may not be applicable to every target tissue and/or chemical. Additionally, this strategy intends to address only the initial steps of the systematic process necessary to synthesize and interpret a mechanistic evidence database, with subsequent aspects focusing on individual study quality evaluations, sensitivity analyses, and weighing of the various evidence components. Nonetheless, we posit that clear definition of the study question, followed by implementation of a comprehensive, integrative, and clear selection and presentation process for mechanistic evidence will result in improved clarity of the scientific support used for risk evaluations related to environmental chemicals.

\section{Methodology}

\subsection{Specification of the study question}

Figure 1 presents a workflow of the systematic approach detailed below. In Step 1, a clearly specified study question is identified to focus the literature search strategy. For this case study, the general question was "What are mechanisms by which chronic exposure to the chemical (DEHP) may cause carcinogenicity in the target tissue (liver)?'”For each mechanistic event, this question was then divided into three subparts. (i) Does the mechanistic event occur with DEHP exposure in humans or cells of human origin? (ii) Does the mechanistic event occur with DEHP exposure in experimental animals including rodents or other species (e.g., non-human primates)? (iii) Does the mechanistic event contribute to DEHP hepatocarcinogenicity? The question, including its subparts, was framed such that the pertinence of the evidence applicable to DEHP, liver cancer and the particular mechanistic event of interest could be evaluated.

The significance of this study question for assessing the overall health hazard of DEHP is based on multiple reports of hepatocarcinogenicity in two rodent species and both sexes upon chronic exposure (Melnick et al., 1987). Additionally, the liver is among the more sensitive target organs in which the carcinogenicity of DEHP has been identified in rodents. Differing conclusions have been reached about the potential relevance of the liver tumor findings to humans. In contrast to the 2000 IARC conclusions regarding the carcinogenic risks of DEHP (IARC, 2000), the 2011 IARC Monographs Working Group evaluating DEHP concluded that "the human relevance of the molecular events leading to DEHPinduced cancer in several target tissues (e.g., liver and testis) in rats or mice could not be ruled out, resulting in the evaluation of DEHP as a Group 2B agent [possibly carcinogenic to humans], rather than Group 3 [not classifiable as to its carcinogenicity to humans]"(Grosse et al., 2011, IARC, 2012). For these reasons, the question of how DEHP may be associated with liver carcinogenicity could be informative of both qualitative and quantitative conclusions in assessing its relevance to human cancer hazard. We focused on the use of a systematic approach for review of the literature database on this important topic.

Similar to the present DEHP example, study questions concerning issues relevant to health hazard decision-making may be raised for other chemicals with voluminous literature in the course of nomination or evaluation for health hazard assessment by EPA, other federal agencies, other organizations, or the public. Target tissue-specific questions may be framed based on detection of the chemical in the human body, indications of human health relevance, or indications of environmental contamination (NRC, 1991). These questions may also be informed by exposure-specific information on human populations at various lifestages. 


\subsection{Identification of relevant mechanisms of action}

Most environmental chemicals are thought to operate through multiple mechanisms and the challenge of comprehensive review in the overall evaluation of mechanistic evidence has been considered (Gehlhaus et al., 2011; Guyton et al., 2008). For this work, we constructed a comprehensive list of likely potential mechanisms of DEHP-induced liver carcinogenesis from reviews on the mechanisms of DEHP liver effects published by a diverse author set (Caldwell, 2012; Guyton et al., 2009; Klaunig et al., 2003; Peters et al., 2005; Roberts et al., 2007; Rusyn and Corton, 2012; Rusyn et al., 2006; Melnick, 2001; McKee, 2000). We considered reviews published since the release of the 2000 IARC monograph on DEHP (IARC, 2000), and selected recent, as well as highly cited older publications.

\subsection{Literature search strategy}

A PubMed (http://www.ncbi.nlm.nih.gov/pubmed/) search strategy was devised to account for mechanistic studies that may have used either the parent compound and/or its metabolites. In the case of DEHP, many studies have used major metabolites (e.g., monoethylhexyl phthalate, MEHP) to account for rapid hydrolysis of the parent molecule in the gastrointestinal tract (Kluwe, 1982). The search was first conducted using only "DEHP" as the term, and the number of results, as well as the medical subject heading (MeSH) decompositions, was recorded and dated (Table 1). To acquire mechanistic evidence for metabolites of DEHP, we created an inclusive string of relevant search terms based on review of recent comprehensive studies of DEHP metabolism in humans and other species (Lorber et al., 2010; Lorber et al., 2011; Svensson et al., 2011; Teitelbaum et al., 2012). The expanded search string was submitted to PubMed and the number of results and MeSH subheadings recorded. MeSH subheadings were manually verified for relevance to the search terms entered. Subheadings deemed irrelevant (e.g., not a known metabolite of DEHP) were deleted from the PubMed query translation, the search was repeated and any changes in results were recorded.

\subsection{Literature searches, mechanisms of action}

The search string for DEHP and its metabolites was expanded to incorporate the target tissue (liver), a particular mechanistic event, and the relevant species of interest to build a literature database of evidence. Based on the comprehensive reviews (see section 2.2), we selected humans, non-human primates and rodents (rat or mouse) as most relevant for the purpose of constructing the evidence tables for DEHP as a case study. We employed wildcards and synonyms for relevant search terms to most effectively identify pertinent literature (Table 1). As described in section 2.3, we recorded and dated PubMed query results, including MeSH subheadings.

\subsection{Application of inclusion/exclusion criteria}

Table 2 lists tiered criteria by which retrieved literature was manually screened by a subject topic expert using the full text research articles for inclusion into evidence tables pertaining to each consensus mechanistic event. The criteria permit transparent and consistent critical evaluation of evidence for tabular presentation. The Tier 1 criteria used in evaluating all of the retrieved literature considered whether the publication addressed the chemical, tissue, mechanism, and species of interest, and whether it contained original data. If a particular mechanism of action had been a subject of many studies (e.g., the number of publications containing original data within a category exceeded 20), additional Tier 2 criteria were applied addressing the study's design and reporting, such as employment of multiple doses, inclusion of at least three biological replicates (the minimum required to conduct a statistical t-test), reporting of experimental variability, and clarity of description of the study's methodology. For this case study, we considered "sufficient replicates" to be at least an N of 
3 based on statistical considerations. These criteria were informed by the US EPA's Guidelines for Carcinogen Risk Assessment (EPA, 2005).

\subsection{Construction of the literature trees}

In the next step, flow diagrams we termed "literature trees" were constructed to visualize and document how initial search findings were manually narrowed to those considered for inclusion into the evidence tables. Beginning from the highest level of the tree, the chemical of interest, publications that were not relevant to the target tissue, selected mechanism, or species were removed. After excluding reviews as determined by PubMed, full text references were then retrieved for manual consideration and inclusion into evidence tables, with Tier 2 assessment criteria applied to large databases.

\subsection{Construction of the evidence tables}

Evidence tables for each potential DEHP mechanism of action in liver were constructed and stratified in accordance with the type of experimental system(s) and other relevant information. Representative examples of the type of information included in these evidence tables are shown in Tables 3, 4, and 5. Data was extracted manually into Microsoft Excel and this program was used to calculate effect as a percent change of control. If a study of interest only presented relevant information in graphical form, the GrabIt! Excel plug-in (www.datatrendsoftware.com) was used for extracting the quantitative information. Results were grouped according to whether studies were conducted in vivo or in vitro, and subsequently sorted with those testing longer duration, lower exposures presented first. Studies that tested the relationship of the mechanistic event to an outcome by evaluating the impact of altering, suppressing or enhancing the mechanism (e.g. in genetically-modified animal models) were prioritized in the presentation. Information about the study could be readily accessed via a hyperlink to the Health and Environmental Research Online (HERO) database (http://hero.epa.gov) provided for each reference. Tables also capture the experimental system or materials used, the number of samples tested, and any relevant treatment periods. Marker(s) or endpoint(s) for each mechanistic event were clearly stated, and quantitative information, where available, on the doses corresponding to the observed changes in that endpoint was provided. Experimental results were expressed as percent change from control and statistical, as reported by the study authors, was indicated. Results for each potential mechanism of action relevant to each species of interest were briefly summarized (in future applications of this strategy, those studies excluded for presentation purposes using Tier 2 criteria may be summarized in this text or in supplemental materials), taking into consideration the types of effects observed, consistency across studies, doseresponse relationships, and the statistical significance of effects reported by the study authors.

\subsection{Literature organization in HERO}

The primary literature considered in the assessment of DEHP mechanisms of action in the liver was uploaded and organized in the HERO database. As appropriate, references were labeled with both the inclusion and exclusion assessment criteria, as well as other pertinent information (e.g., species), to enhance transparency and to facilitate navigation and searching.

\section{Results}

\subsection{Literature search for DEHP and its metabolites}

The initial search performed by entering "DEHP" into PubMed yielded a return of 2319 references (as of June 25, 2012), with MeSH subheadings all relevant to DEHP and other 
phthalates (Table 1 and Figure 2). However, some in vitro mechanistic studies may have been conducted with DEHP metabolites, not the parent compound. Accordingly, an expanded search string including metabolites and relevant chemical abbreviations and prefixes (see Table 1) yielded 3361 publications (as of June 25, 2012), an increase of nearly $45 \%$. The MeSH query translations were manually examined and terms not relevant to DEHP metabolism (e.g., "2-methyl-5,6-cyclopentapyrimidine") were excluded. Exclusion of such headings did not affect the number or content of search results returned.

\subsection{Identification of DEHP mechanisms of action in liver}

Based on a survey of seven recent and/or highly cited reviews from diverse (in terms of affiliation with the government, academia and/or industry sectors) authorship (Caldwell, 2012; Guyton et al., 2009; Klaunig et al., 2003; Peters et al., 2005; Roberts et al., 2007; Rusyn and Corton, 2012; Rusyn et al., 2006, Melnick, 2001; McKee, 2000) nine mechanistic events for DEHP in liver were identified (Figure 2). These were peroxisome proliferation, cell proliferation, PPAR receptor activation, oxidative stress, nuclear receptor activation, genotoxicity, apoptosis, disruption of gap junction intercellular communication, and Kupffer cell activation. Accordingly, search phrases describing the tissue of interest, liver, and each of the mechanistic events were composed and concatenated with the search string devised for DEHP and its metabolites. Query translation anomalies were addressed, and irrelevant MeSH headings were also removed. For instance, because the MeSH query translation for liver did not include the synonym "hepatic" or any variation thereof, the wildcard "hepat*" was included to maximize returns for relevant evidence (Table 1). An example search string for one of the mechanistic events (peroxisome proliferation) is also shown in Table 1. Each search string and the corresponding MeSH headings used in the final search were saved to enable future examination of the search terms used.

\subsection{Application of inclusion/exclusion criteria, construction of literature trees and evidence tables}

After collecting literature lists for each of the mechanistic events, we subjected each reference to predefined assessment criteria (Table 2) to determine its suitability for inclusion into an evidence table. As recommended by the NRC (2011), a literature tree was created for each of the mechanistic events to graphically document inclusion/exclusion criteria and the number of publications kept or removed at each step (Figures 3-5). Here, we provide several examples illustrating the process and the format for the information entered into the evidence tables.

"Peroxisome proliferation" is a mechanistic event with the largest literature database, where 358 publications were identified by the PubMed search (Figure 3 and Table 1). Upon inclusion of species-specific search terms (Table 1), most references were subdivided as relevant to humans, rodents, and non-human primates. Studies reporting results in other species were also noted, totaling 4 references to DEHP toxicity studies in rabbits or fish. Reviews, as identified by PubMed, were not considered further.

Next, the articles were considered for inclusion by manually examining the abstracts of each study. As an example, of the 324 publications identified by the PubMed query as containing information on peroxisome proliferation in rodents, 262 were excluded, most because they were not studies of the relevant chemical (DEHP or its metabolites), tissue (liver), species (rodents), or the mechanism of interest. Many lacked primary data, but were not indexed as reviews by PubMed. This first manual review step was essentially a reapplication of the Tier 1 assessment criteria. A follow-up step considered the full text of each remaining publication according to Tier 2 criteria (Table 2) and 43 additional studies were excluded for reasons identified in Figure 3. Ultimately, 19 unique publications were selected for inclusion 
into the evidence table for peroxisome proliferation in rodents. Table 3 shows representative tabular formatting of some of the experimental evidence. For each mechanistic event, a table may be subdivided by species and type of study (in vivo or in vitro), with further categorization of in vivo studies by strain, sex and study duration (chronic cancer bioassay, subchronic or acute).

"Peroxisome proliferator activated receptor" is a mechanistic event with an intermediatesized literature database, where 46 publications were identified by the PubMed search (Figure 4). Interpretation of this mechanistic event in the human health assessment of a number of peroxisome proliferators has been controversial due to differing views as to its relevance to humans (Klaunig et al., 2003; Melnick, 2001). Several studies that addressed this mechanism of liver carcinogenesis have challenged the hypothesized mode of action using knockout and transgenic (e.g., humanized) mouse strains. Table 4 provides several representative examples of how evidence from such studies can be displayed.

"Gap junction intercellular communication" (GJIC) is representative of a mechanistic event with a small, yet potentially important, literature database (Figure 5). Only 12 articles were identified for GJIC. Each reference was assessed using the PubMed search through Tier 1 criteria, and a breakdown of the amount of evidence for each species of interest and whether or not the reference contained original data was performed. At the end, 1 unique article for humans, 5 for rodents, and 2 for non-human primates were selected for inclusion into the evidence table for GJIC which is included in its entirety as an example (Table 5).

\subsection{Short narrative summary for mechanisms of action}

Succinct summaries reporting critical conclusions from the gathered evidence, and capturing details relevant to study design, observed effects, dose-response relationships, and consistency across various research groups can readily be generated from the evidence tables. Concordance of the results across species may be considered here, given completion of study selection and presentation. The paragraphs that follow provide an example of a short narrative that can be developed from the evidence table for GJIC (provided in its entirety as Table 5).

"In humans or other primates, no significant in vitro effects on GJIC were observed in a single study of human hepatocytes, in a single human cell line, or in a single study of nonhuman primate hepatocytes. Similarly, a single dose in vivo study in male primates did not observe effects on GJIC following short-term treatment. No human or non-human primate studies evaluated females or exposures longer than two weeks. The only measure of GJIC used in these studies was in situ dye transfer (ISDT).

In rodents, only a small body of evidence suggests that chronic exposure to DEHP disrupts GJIC. A statistically significant decrease in dye transfer of about $40 \%$ was observed in male F344 rats after short term exposure to DEHP, and non-statistically significant decreases of approximately $30 \%$ were observed in a single study of male rats and mice treated for 18 months. In vitro, dose-dependent significant decreases were observed across four studies examining primary hepatocytes derived from male and female mice and two strains of male rats, following treatments with DEHP or MEHP for up to six weeks. The only measure of GJIC used in these studies was ISDT. Decreases in GJIC following short-term DEHP treatment were consistent and dose-related across several studies with differing sexes, species, or strains. 


\section{Discussion}

The goal of this study was to develop and implement a systematic approach for literature review, identification and presentation that would improve transparency of evidence collection and standardization of reporting for human health assessment of chemical hazards. The process detailed herein addresses critical points of guidance presented in recent NRC reports (NRC, 2010; NRC, 2011). Specifically, we focused on the protocol for standardization of the literature review through a systematic and transparent process, and developed a template for presentation of reviewed studies in tabular and graphic form so that the key dimensions of study characteristics and pertinence are clear.

A major benefit of this work includes reducing a large literature database into a smaller, more manageable one, allowing for better analysis of the strength of evidence provided by concordance of results across studies, species, strain, sex, dose and time of exposure as well as endpoint assessment. The strategy has also proven effective for appraisal and synthesis of large amounts of information, and is conducive towards visual data displays that help track capture of results and thus facilitate decision-making processes. The workflow in the process presented herein is also iterative such that the results from each previous step are carried forward and built upon in the subsequent steps.

Several key features of our process directly address elements common to systematic evidence review and evaluation. First, we selected, and provide transparent documentation of, search terms used to identify literature describing mechanisms of action of DEHP and its metabolites. Incorporating synonyms and wildcards representing critical terms in the PubMed search is necessary in order to obtain the most comprehensive results possible.

Second, establishment of tiered assessment criteria for inclusion and exclusion of literature allows for transparent and consistent critical evaluation of evidence for tabular presentation. These criteria focus on study relevancy to the predefined research question and experimental design. The tiered approach also effectively reduces large literature databases to manageable ones, facilitating extraction of data for inclusion in evidence tables. As applied to selecting pertinent mechanistic evidence for DEHP, our criteria included consideration of target tissues, explicit identification of species and sex of animals, whether the study was in vitro or in vivo, dosing information, and characteristic endpoints of the relevant mechanisms. These were applied in the determination of the pertinence of the studies to the predefined research question. We envision that subsequent steps of the evaluation could systematically assess bias and study quality, and evaluate weight-of-evidence based upon the literature collected and screened.

Third, visualization of the evidence inclusion and exclusion in the form of a flow diagram, or literature tree, comprehensively documents study selection. The progression from the initial 3361 articles identified as relevant to DEHP to those included in the mechanismbased evidence tables is transparent, displaying how search findings are narrowed to articles in the final review selection based upon the pre-defined criteria.

Fourth, by summarizing findings in evidence tables, the need for long descriptions of studies for each mechanism is effectively eliminated. The tables include information about the publication, as well as necessary experimental details and dose-response relationships, if any. The results of statistical analyses of the findings by the authors, where applicable, were noted. Additionally, we constructed tables in a manner that highlights significant results and consistency of dose-response relationships in each species of interest. This is especially useful to the mechanistic evidence evaluation, as US EPA guidance (EPA, 2005) indicates that the strongest mechanistic evidence is provided by studies that experimentally challenge the role of the mechanism in carcinogenesis or other adverse outcomes, in particular by 
evaluating the impact of altering, suppressing or enhancing the mechanism (e.g., in genetically-modified or other experimental models, or in recovery experiments). The experimental evidence included in the table of the peroxisome proliferation mechanism provides an example addressing this critical point. Furthermore, the tabular presentation can highlight the extent of support across experimental designs, consistent with US EPA guidance (EPA, 2005) that conclusions are meaningfully strengthened by consistent results in different experimental models, but not by replicate experiments in the same model. Finally, as illustrated for GJIC disruption (for which the evidence tables is provided in entirety in Table 5), short narratives summarizing the presented evidence can readily be developed from the tables.

This stepwise process is also potentially informative for quantitative aspects of chemical hazard assessment, including ranking of evidence for applicability to dose-response analysis, or in the conduct of meta-analyses across studies, chemicals, or species. We illustrate comparability of study results based upon experimental characteristics rather than the direction or magnitude of outcomes, consistent with the Navigation Guide Project supporting evidence-based decision-making in environmental health (Woodruff and Sutton, 2011). The NRC (NRC, 2011) also suggested that IRIS assessments document the rationale by which studies are excluded or included for derivation of reference values for noncancer endpoints and unit risks for cancer endpoints. Systematic approaches for evidence identification and presentation may facilitate assessment of the experimental conditions and quantitative outcomes within the referenced body of evidence. From this, we anticipate that a formal assessment of consistency across studies, displays of variability (e.g., 95\% confidence intervals), as well as assessments of study quality, could be performed as a next step in the evaluation.

There are, however, limitations identified through the conduct of this study. To ensure the transparency and reproducibility of search strategies, we utilized a publicly available database (i.e., PubMed) for identification of the evidence. Although this database is extensive, searching it exclusively may nonetheless miss studies; thus, one of the lessons learned applicable to future literature identification projects is the need to assess the benefit of extending the search to other online databases (e.g., ToxNet, Web of Science, technical study reports, or foreign-language pertinent literature sources). As an example, a search for "DEHP and peroxisome proliferator activated receptor" using the Web of Science database interface yielded over twice the number of references than retrieved from PubMed alone (data not shown). The relative merits of systematically capturing mechanistic information from "grey" literature (e.g., technical reports) or restricted-access evidence materials (e.g., proprietary information submitted for regulatory review by commercial entities) also need to be explored.

Additionally, we did not attempt to retrieve studies identifying novel mechanistic events of DEHP that had not been the subject of prior published reviews. While anticipated to be few in numbers, those additional pertinent documents may be identified using broader search strategies. The retrieved results can be filtered against the combined reference lists of the published reviews to aid in identifying new mechanistic events and their associated search terms. Finally, our literature search terms captured only DEHP-specific studies. Thus, readacross from other chemicals (e.g., other peroxisome proliferators), tissues and life-stages that may have an even more comprehensive database would not be picked up by our process which makes it difficult to tackle issues such as biological plausibility or analogy to other chemicals.

Our approach also underscores the importance of manual expert review. We found that computerized database searches both excluded references with relevant experimental data 
and included those without primary research results (e.g., review articles). For example, we identified articles that contained chemical-relevant experimental results that are not explicitly described in the study abstract or methods. We also found that search strings employed to gather the literature were not necessarily exclusive at the level of species. For example, a study may repeatedly reference human data in the introduction and/or discussion, but experimentally present rodent data. Additionally, because PubMed does not index all reviews, our searches retrieved some articles that lacked original data. These examples highlight the need for manual expert review of full text and figures, with application of inclusion and exclusion criteria to determine the relevance of results for extraction.

We employed a quality control process involving subsequent review by one additional expert in order to ensure correct categorization of the retrieved articles. However, in keeping with the best practices of systematic review, we would further strengthen our approach in the future by utilizing two independent screeners and outlining clear strategies at the outset for resolving any conflicts encountered during critical examination of the pertinence of the identified evidence. Our effort could also be extended to the subsequent aspects of systemic evidence appraisal including assessment of bias and evaluation of weight-of-evidence. Given time and resource constraints, the present effort focused on information search, selection, and presentation, addressing about half of the key practices of systematic review as outlined by the Cochrane Collaboration (2006).

In summary, by employing computerized retrieval of literature in PubMed, expert consideration of articles for inclusion, and methods of evidence reporting that can be conducted and displayed in a consistent and transparent manner, we have developed an approach that will strengthen the validity of scientific evidence evaluations in chemical hazard assessments. The resulting deliverables can also be included in various portions of an IRIS Toxicological Review, with evidence tables and short narrative summaries in the primary body of the document, and search strategies, literature trees, and assessment criteria included in an appendix. Online resources such as the HERO database can also be utilized to capture study information, with all of the literature considered in the assessment classified in accordance with inclusion and exclusion criteria. Our process also allows for expert evaluation of results at every stage, a critical component to conduct an interpretation of the relevance of results to the assessment process. Ultimately, this systematic approach to literature retrieval, selection, and presentation of studies effectively manages a large volume of literature, improves transparency, and facilitates synthesis of critical information (e.g., regarding hepatocarcinogenicity mechanism of action) in human health assessments.

\section{Acknowledgments}

We thank Dr. Babasaheb Sonawane for management support of this work. Mary Kushman and Ivan Rusyn were supported, in part, by the Oak Ridge Institute for Science and Education fellowships made available through funding from the US Environmental Protection Agency. This work was also supported, in part, by the National Institutes of Health grant P42 ES005948.

\section{References}

Caldwell JC. DEHP: genotoxicity and potential carcinogenic mechanisms-a review. Mutat Res. 2012; 751:82-157. [PubMed: 22484601]

David RM, et al. Chronic peroxisome proliferation and hepatomegaly associated with the hepatocellular tumorigenesis of di(2-ethylhexyl)phthalate and the effects of recovery. Toxicol.Sci. 1999; 50:195-205. [PubMed: 10478855]

DHHS. The Health Consequences of Smoking. A report of the Surgeon General. 2004 
Drummond MB, et al. Inhaled corticosteroids in patients with stable chronic obstructive pulmonary disease: a systematic review and meta-analysis. JAMA. 2008; 300:2407-2416. [PubMed: 19033591]

EPA. Guidelines for carcinogen risk assessment. 2005

Eveillard A, et al. Di-(2-ethylhexyl)-phthalate (DEHP) activates the constitutive androstane receptor (CAR): a novel signalling pathway sensitive to phthalates. Biochem Pharmacol. 2009; 77:17351746. [PubMed: 19428328]

Feige JN, et al. The pollutant diethylhexyl phthalate regulates hepatic energy metabolism via speciesspecific PPARalpha-dependent mechanisms. Environ Health Perspect. 2010; 118:234-241. [PubMed: 20123618]

Gehlhaus MW, 3rd, et al. Approaches to cancer assessment in EPA's Integrated Risk Information System. Toxicol Appl Pharmacol. 2011; 254:170-180. [PubMed: 21034767]

Goll V, et al. Comparison of the effects of various peroxisome proliferators on peroxisomal enzyme activities, DNA synthesis, and apoptosis in rat and human hepatocyte cultures. Toxicology \& Applied Pharmacology. 1999; 160:21-32. [PubMed: 10502499]

Grosse Y, et al. Carcinogenicity of chemicals in industrial and consumer products, food contaminants and flavourings, and water chlorination byproducts. Lancet Oncology. 2011; 12:328-329. [PubMed: 21598447]

Guyatt GH, et al. Experience with GRADE. J Clin Epidemiol. 2012a; 65:1243-1244. [PubMed: 22981248]

Guyatt GH, et al. GRADE guidelines-an introduction to the 10th-13th articles in the series. J Clin Epidemiol. 2012b

Guyatt GH, et al. GRADE guidelines: a new series of articles in the Journal of Clinical Epidemiology. J Clin Epidemiol. 2011; 64:380-382. [PubMed: 21185693]

Guyton KZ, et al. Mode of action frameworks: a critical analysis. J Toxicol Environ Health B Crit Rev. 2008; 11:16-31. [PubMed: 18176885]

Guyton KZ, et al. A reexamination of the PPAR-alpha activation mode of action as a basis for assessing human cancer risks of environmental contaminants. Environ Health Perspect. 2009; 117:1664-1672. [PubMed: 20049115]

Hinton RH, et al. Effects of phthalic acid esters on the liver and thyroid. Environmental Health Perspectives. 1986; 70:195-210. [PubMed: 3830106]

IARC, Di(2-ethylhexyl) phthalate. Some industrial chemicals. Vol. Vol. 77. Lyon, France: 2000. p. 41-148.

IARC, Di(2-ethylhexyl)phthalate. Some chemicals present in industrial and consumer products, food, and drinking-water. Vol. Vol. 101. Lyon, France: 2012. p. 149-284.

IOM. Improving the presumptive disability decision-making process for veterans. Washington DC: 2008.

Isenberg JS, et al. Reversibility and persistence of di-2-ethylhexyl phthalate (DEHP)- and phenobarbital-induced hepatocellular changes in rodents. Toxicol.Sci. 2001; 64:192-199. [PubMed: 11719701]

Isenberg JS, et al. Effects of Di-2-ethylhexyl phthalate (DEHP) on gap-junctional intercellular communication (GJIC), DNA synthesis, and peroxisomal beta oxidation (PBOX) in rat, mouse, and hamster liver. Toxicol.Sci. 2000; 56:73-85. [PubMed: 10869455]

Ito Y, et al. Plasticizers May Activate Human Hepatic Peroxisome Proliferator-Activated Receptor alpha Less Than That of a Mouse but May Activate Constitutive Androstane Receptor in Liver. PPAR Res. 2012:201284. [PubMed: 22792086]

Ito Y, et al. Di(2-ethylhexyl)phthalate induces hepatic tumorigenesis through a peroxisome proliferator-activated receptor alpha-independent pathway. J Occup Health. 2007; 49:172-182. [PubMed: 17575397]

Kamendulis LM, et al. Comparative effects of phthalate monoesters on gap junctional intercellular communication and peroxisome proliferation in rodent and primate hepatocytes. $\mathrm{J}$ Toxicol.Environ.Health A. 2002; 65:569-588. [PubMed: 11995694]

Klaunig JE, et al. PPARalpha agonist-induced rodent tumors: modes of action and human relevance. Crit Rev.Toxicol. 2003; 33:655-780. [PubMed: 14727734] 
Klaunig JE, et al. Inhibition of mouse hepatocyte intercellular communication by phthalate monoesters. Cancer Letters. 1988; 43:65-71. [PubMed: 3203332]

Kluwe WM. Overview of phthalate ester pharmacokinetics in mammalian species. Environ Health Perspect. 1982; 45:3-9. [PubMed: 6754362]

Leibold $\mathrm{E}$, et al. Inhibition of intercellular communication of rat hepatocytes by nafenopin: involvement of protein kinase C. Carcinogenesis. 1994; 15:1265-1269. [PubMed: 8020165]

Lorber M, et al. A simple pharmacokinetic model to characterize exposure of Americans to di-2ethylhexyl phthalate. J Expo Sci Environ Epidemiol. 2010; 20:38-53. [PubMed: 19127283]

Lorber M, et al. A critical evaluation of the creatinine correction approach: can it underestimate intakes of phthalates? A case study with di-2-ethylhexyl phthalate. J Expo Sci Environ Epidemiol. 2011; 21:576-586. [PubMed: 21289653]

McKee RH. The Role of Inhibition of Gap Junctional Intercellular Communication in Rodent Liver Tumor Induction by Phthalates: Review of Data on Selected Phthalates and the Potential Relevance to Man. Regul Toxicol Pharmacol. 2000; 32:51-55. [PubMed: 11029268]

Melnick RL. Is peroxisome proliferation an obligatory precursor step in the carcinogenicity of di(2ethylhexyl)phthalate (DEHP)? Environmental Health Perspectives. 2001; 109:437-442. [PubMed: 11401753]

Melnick RL, et al. Studies by the National Toxicology Program on di(2- ethylhexyl)phthalate. Toxicol Ind Health. 1987; 3:99-118. [PubMed: 3617073]

NRC. Monitoring Human Tissues for Toxic Substances. Washington DC: 1991.

NRC. Science and Decisions: Advancing Risk Assessment. Washington DC: 2009. p. 424

NRC. Review of the Environmental Protection Agency's Draft IRIS Assessment of Tetrachloroethylene. 2010

NRC. Review of the Environmental Protection Agency's Draft IRIS Assessment of Formaldehyde. Washington DC: 2011.

Peters JM, et al. Peroxisome proliferator-activated receptor-alpha and liver cancer: where do we stand? J Mol Med (Berl). 2005; 83:774-785. [PubMed: 15976920]

Pugh G Jr, et al. Effects of di-isononyl phthalate, di-2-ethylhexyl phthalate, and clofibrate in cynomolgus monkeys. Toxicol.Sci. 2000; 56:181-188. [PubMed: 10869467]

Roberts RA, et al. Role of the Kupffer cell in mediating hepatic toxicity and carcinogenesis. Toxicol Sci. 2007; 96:2-15. [PubMed: 17122412]

Rusyn I, Corton JC. Mechanistic considerations for human relevance of cancer hazard of di(2ethylhexyl) phthalate. Mutat Res. 2012; 750:141-158. [PubMed: 22198209]

Rusyn I, et al. Modes of action and species-specific effects of di-(2-ethylhexyl)phthalate in the liver. Crit Rev Toxicol. 2006; 36:459-479. [PubMed: 16954067]

Stroup DF, et al. Meta-analysis of observational studies in epidemiology: a proposal for reporting. Meta-analysis Of Observational Studies in Epidemiology (MOOSE) group. JAMA. 2000; 283:2008-2012. [PubMed: 10789670]

Svensson K, et al. Phthalate exposure associated with self-reported diabetes among Mexican women. Environ Res. 2011; 111:792-796. [PubMed: 21696718]

Teitelbaum SL, et al. Associations between phthalate metabolite urinary concentrations and body size measures in New York City children. Environ Res. 2012; 112:186-193. [PubMed: 22222007]

van der Worp HB, et al. Can animal models of disease reliably inform human studies? PLoS Med. 2010; 7:e1000245. [PubMed: 20361020]

Woodruff TJ, Sutton P. An evidence-based medicine methodology to bridge the gap between clinical and environmental health sciences. Health Aff (Millwood). 2011; 30:931-937. [PubMed:

21555477] 


\section{HIGHLIGHTS}

A systematic approach to literature retrieval and inclusion/exclusion was developed Graphical presentation of mechanistic evidence is demonstrated Di(2-ethylhexyl)phthalate was used as a case study chemical A complex mechanistic evidence database of $>3000$ publications was effectively managed 


\section{Specify the study question}

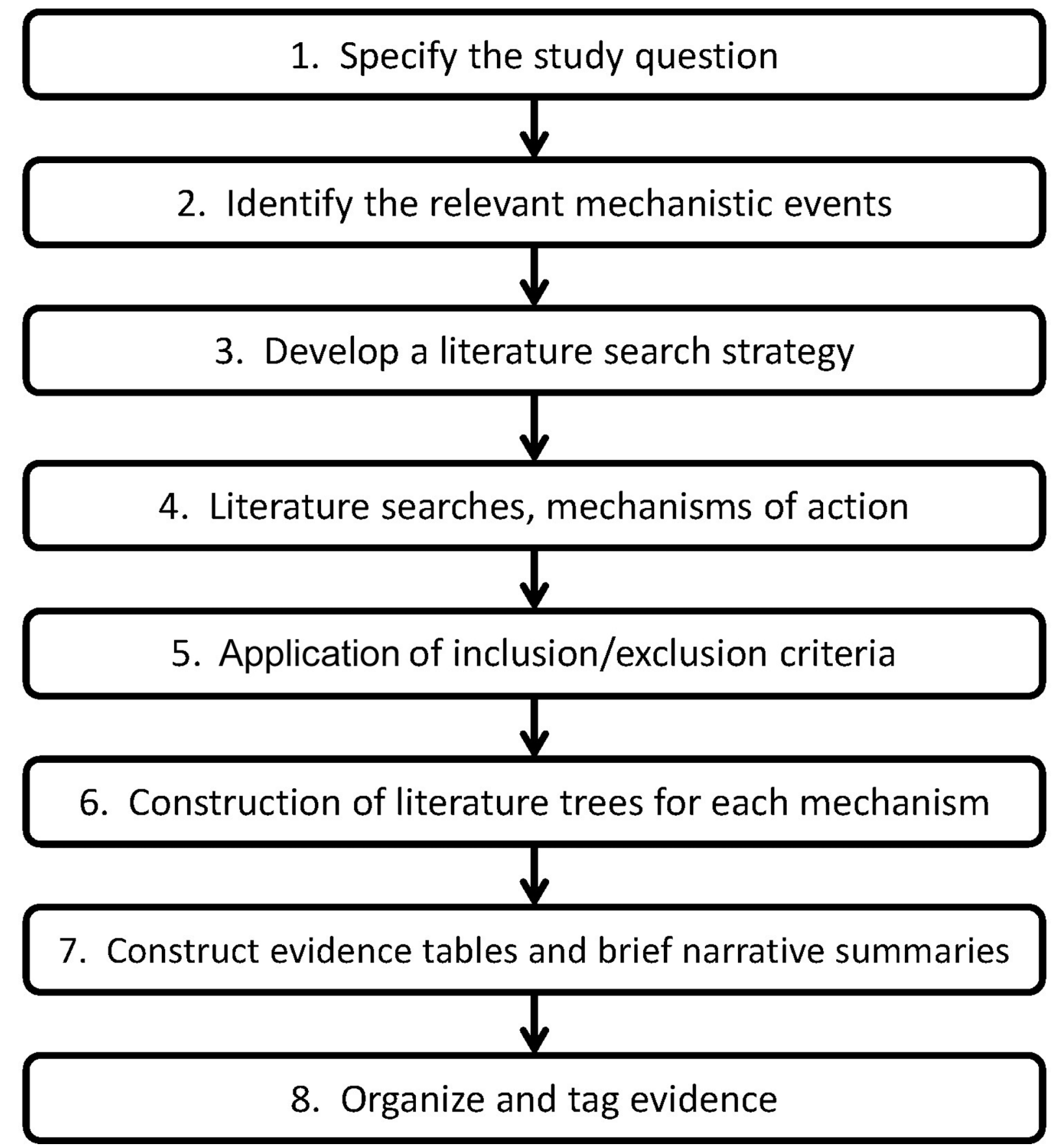

Figure 1.

The 8-step systematic approach for identifying, evaluating, and presenting mechanism of action evidence. 


\section{Specify study Effects of DEHP and its question: metabolites on the liver}

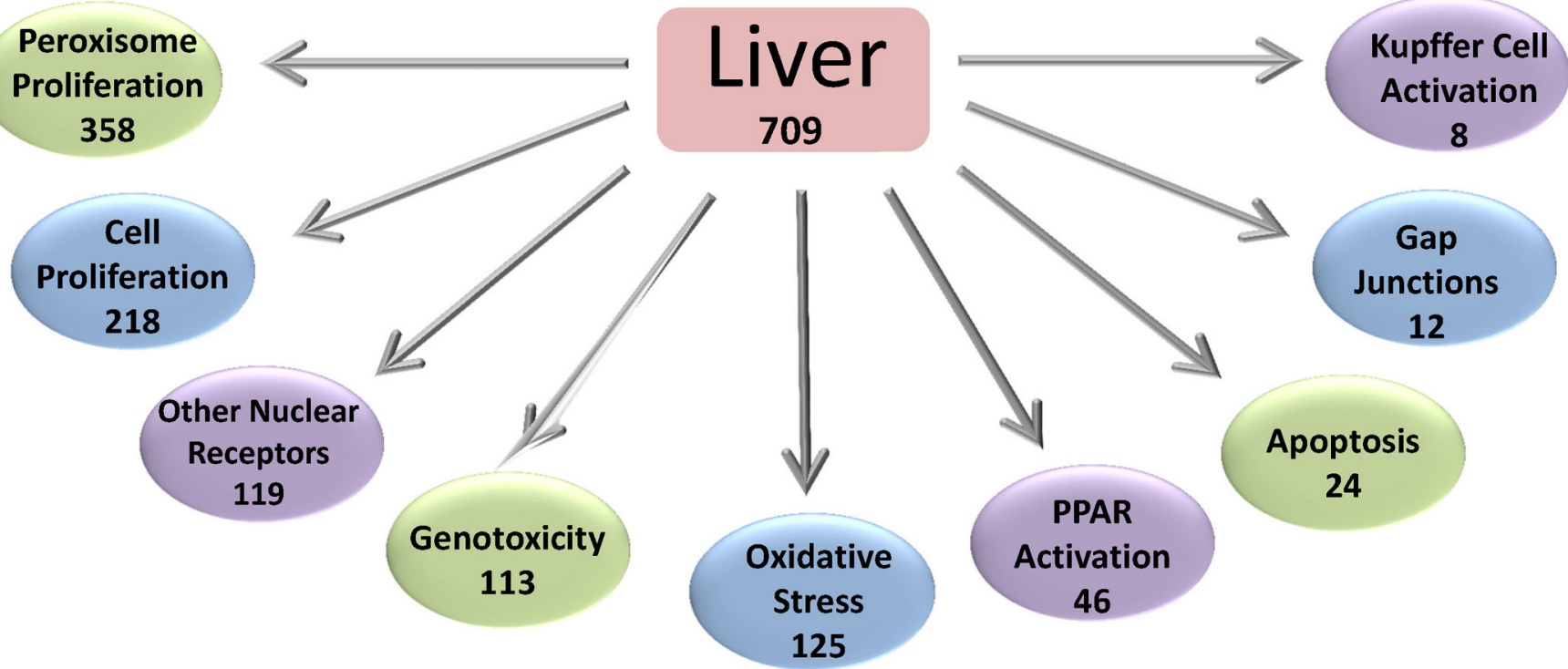

Figure 2.

Consensus mechanistic events elicited by DEHP in liver and PubMed search results for sizes of literature databases for each. The numbers represent the quantity of articles in the databases at the level of chemical, tissue, and mechanisms of action. 
3361 Potentially relevant published articles for "DEHP or its metabolites"

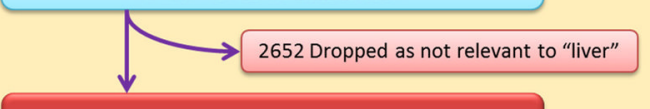

709 Further screened for mechanism of interest

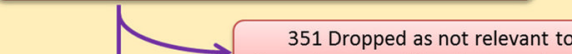
"peroxisome proliferation" mechanism

\section{Further screened for species of interest}

74 published articles in "human" 27 Dropped (review)

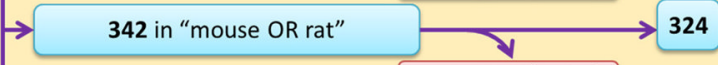

59 in "non-human primate"
18 Dropped (review)
Evaluation of the evidence by an expert:

324 Publications selected for manual evaluation and inclusion into evidence tables

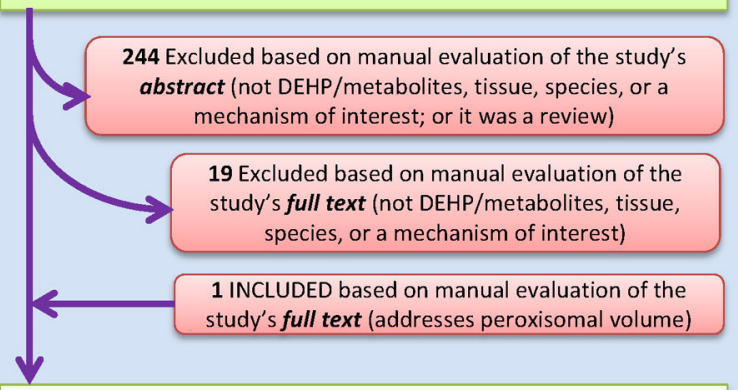

62 Publications screened as full text for possible exclusion

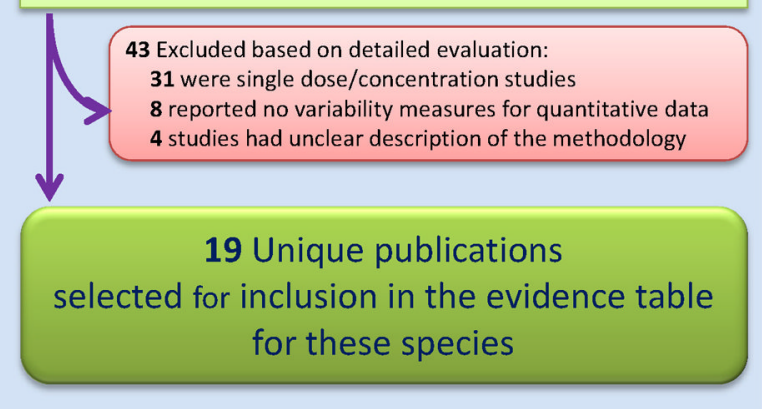

Figure 3.

Literature tree for the "peroxisome proliferation" mechanistic event. Progression from the initial 3361 identified articles relevant to DEHP or its metabolites to those included in the evidence tables is shown. 


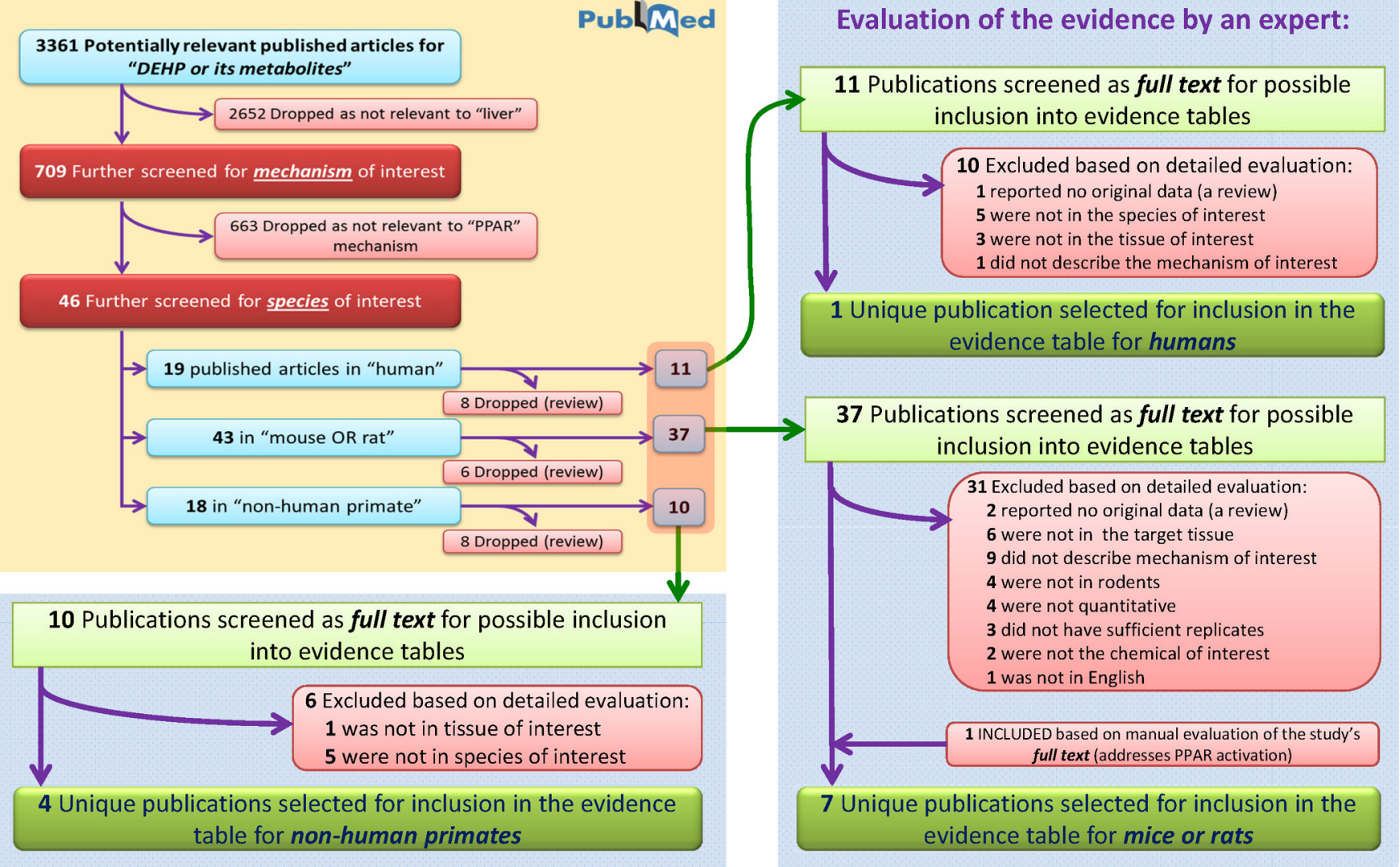

Figure 4.

Literature tree for the "peroxisome proliferator activated receptor" mechanistic event.

Progression from the initial 3361 identified articles relevant to DEHP or its metabolites to those included in the evidence tables is shown. 
3361 Potentially relevant published articles for "DEHP or its metabolites"

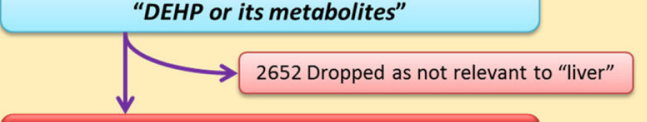

709 Further screened for mechanism of interest

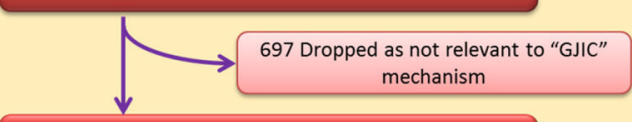

12 Further screened for species of interest

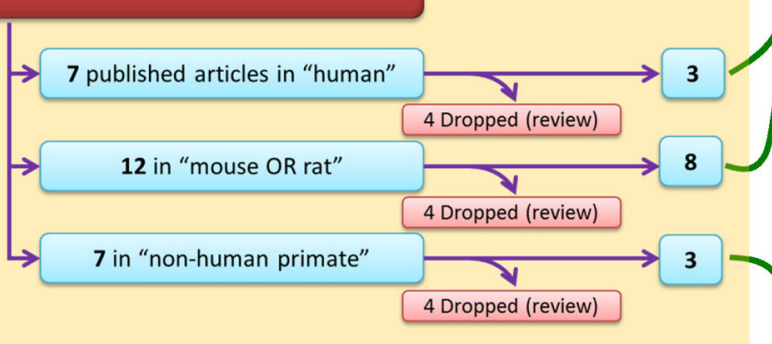

Evaluation of the evidence by an expert:

3 Publications screened as full text for possible inclusion into evidence tables

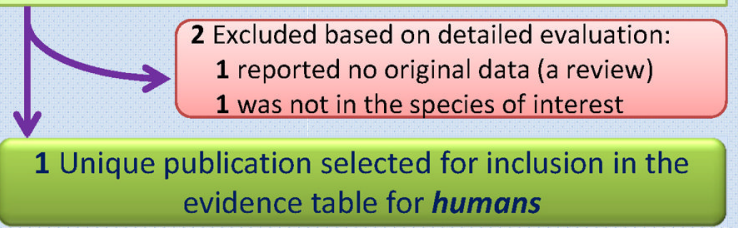

8 Publications screened as full text for possible inclusion into evidence tables

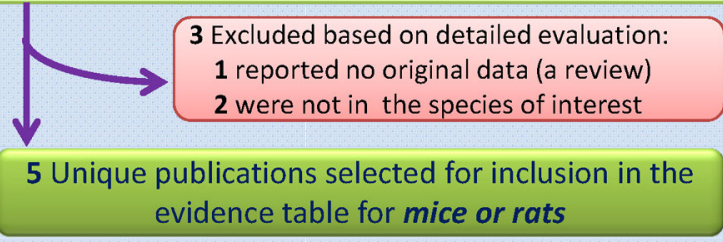

3 Publications screened as full text for possible inclusion into evidence tables

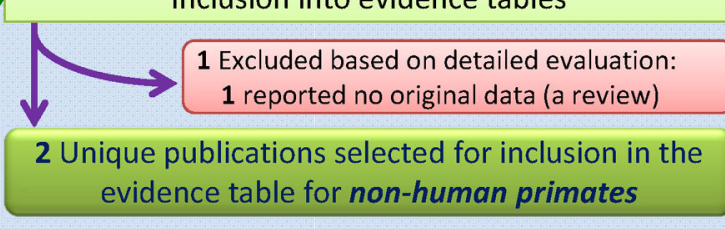

Figure 5.

Literature tree for the "gap junction intercellular communication" mechanistic event.

Progression from the initial 3361 identified articles relevant to DEHP and its metabolites to those included in the evidence tables is shown. 
Table 1

Overall search strategy for DEHP, its metabolites, tissue, and representative mechanism of action and species. Search strings, PubMed MeSH query translations, and returned results for DEHP and its metabolites, the target tissue, selected mechanisms of action, and species of interest. Searches were performed on June 25, 2012.

\begin{tabular}{|c|c|c|}
\hline $\begin{array}{l}\text { Level of } \\
\text { search }\end{array}$ & $\begin{array}{l}\text { Search term } \\
\text { (user query) }\end{array}$ & MeSH query translation \\
\hline Chemical & DEHP & $\begin{array}{l}\text { "diethylhexyl phthalate"[MeSH Terms] OR ("diethylhexyl”[All Fields] AND } \\
\text { "phthalate"[All Fields]) OR "diethylhexyl phthalate"[All Fields] OR "dehp"[All } \\
\text { Fields] }\end{array}$ \\
\hline $\begin{array}{l}\text { Chemical } \\
\text { and its } \\
\text { metabolites }\end{array}$ & $\begin{array}{l}\text { (mono (3- carboxypropyl) OR } \\
\text { mcpp OR mono (2- ethylhexyl) OR } \\
\text { mono (2-ethyl-5- oxohexyl) OR } \\
\text { meohp OR mono } \\
\text { (2-ethyl-5- carboxypentyl) OR } \\
\text { mecpp OR mono } \\
\text { (2-ethyl-5- hydroxyhexyl) OR } \\
\text { mehp OR dehp OR } \\
\text { 2-ethylhexanol OR } \\
\text { (phthalic acid) OR } \\
\text { (2-ethyl-5- hydroxyhexyl) OR } \\
\text { MEHHP OR (2- carboxymethyl) OR } \\
\text { MECPP OR MMHP } \\
\text { OR (2-ethyl-5- oxyhexyl)) }\end{array}$ & 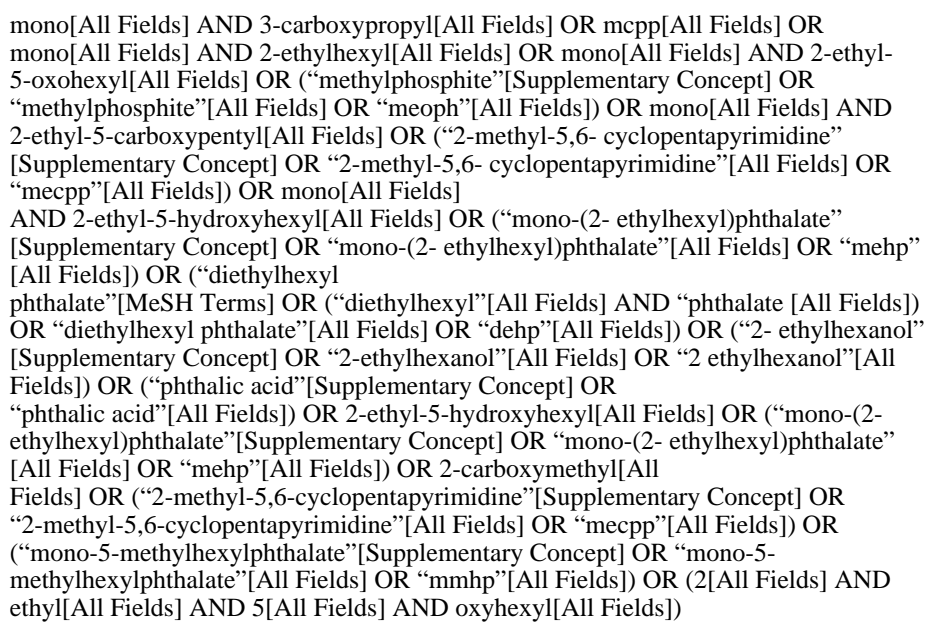 \\
\hline \multirow[t]{2}{*}{ Tissue } & (liver) & liver[MeSH Terms] OR “liver”[All Fields] \\
\hline & (liver OR hepat ${ }^{*}$ ) & (“liver”[MeSH Terms] OR “liver”[All Fields]) OR (hepat[All Fields] OR II...) \\
\hline \multirow[t]{2}{*}{ Mechanism } & (peroxisome proliferation) & $\begin{array}{l}\text { (“peroxisomes”[MeSH Terms] OR “peroxisomes”[All Fields] OR } \\
\text { "peroxisome”[All Fields]) AND proliferation[All Fields] }\end{array}$ \\
\hline & (peroxis ${ }^{*}$ ) & $\begin{array}{l}\text { peroxis[All Fields] OR peroxiscan[All Fields] OR peroxisides[All Fields] OR } \\
\text { peroxismal[All Fields] OR peroxisme[All Fields] OR peroxismes[All Fields] OR } \\
\text { peroxismose[All Fields] OR peroxisom[All Fields] OR peroxisoma[All Fields] OR } \\
\text { peroxisomal[All Fields] OR }{ }^{*} \ldots\end{array}$ \\
\hline \multirow[t]{2}{*}{ Species } & (mouse or rat) & $\begin{array}{l}\text { (“mice”[MeSH Terms] OR “mice”[All Fields] OR “mouse"[All Fields]) OR rat[All } \\
\text { Fields] }\end{array}$ \\
\hline & $\begin{array}{l}\text { (mouse OR rat OR } \\
\text { hamster OR rodent } \\
\text { OR murine OR Mus } \\
\text { musculus or } \\
\text { Rattus) }\end{array}$ & 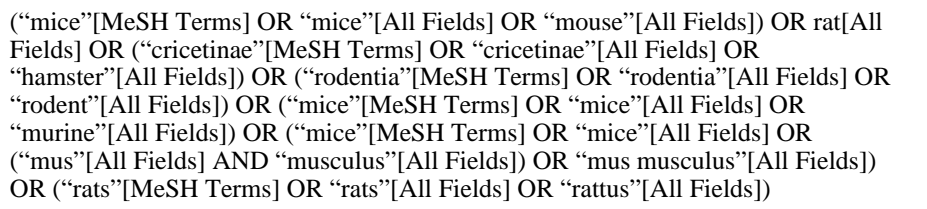 \\
\hline
\end{tabular}




\section{Table 2}

Evaluation criteria used in the consideration of inclusion and exclusion of evidence for DEHP mechanisms of action in this case study.

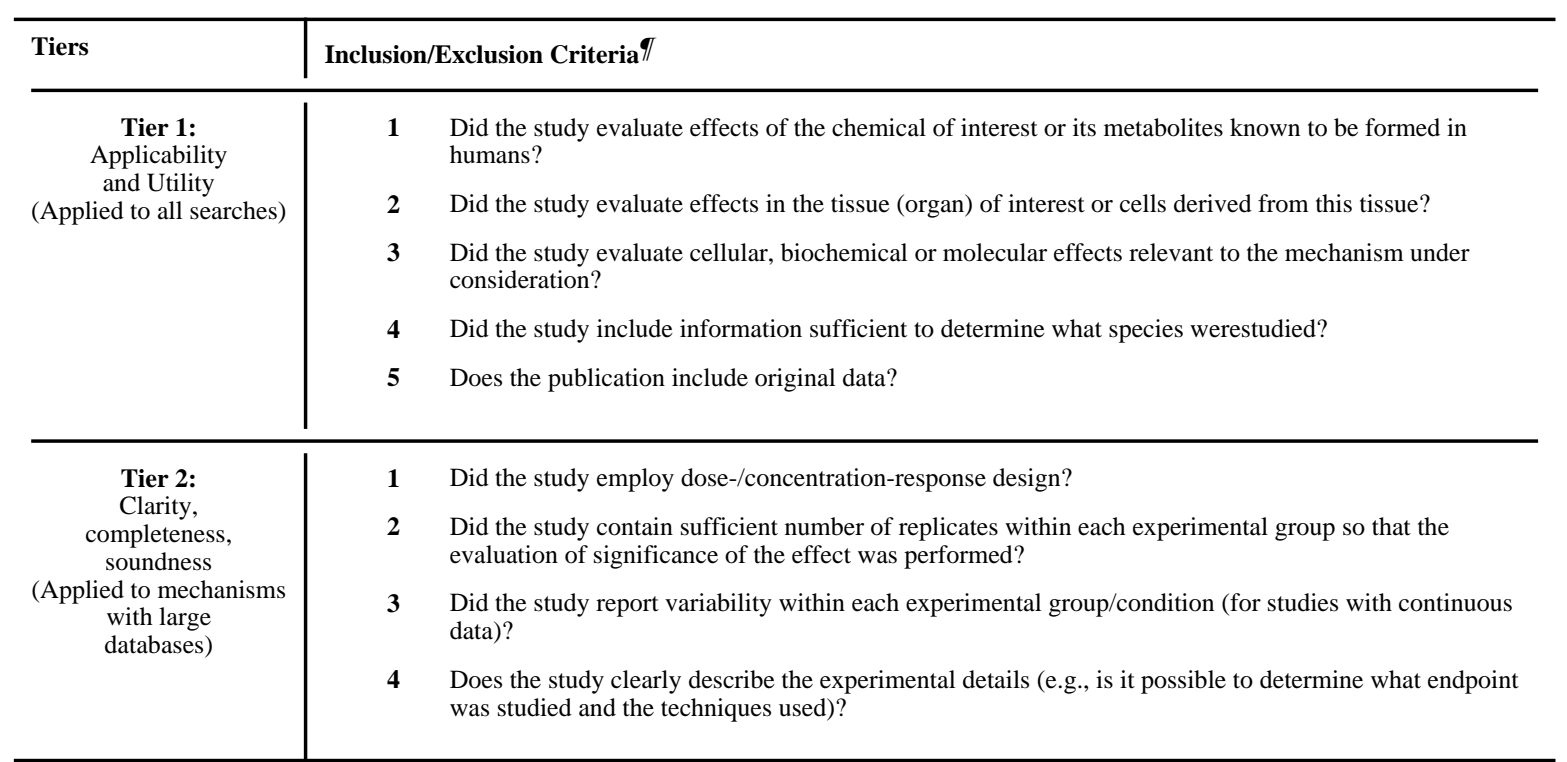

If the answer is "no" to any of these criteria questions, the study will be excluded from the evidence table. 


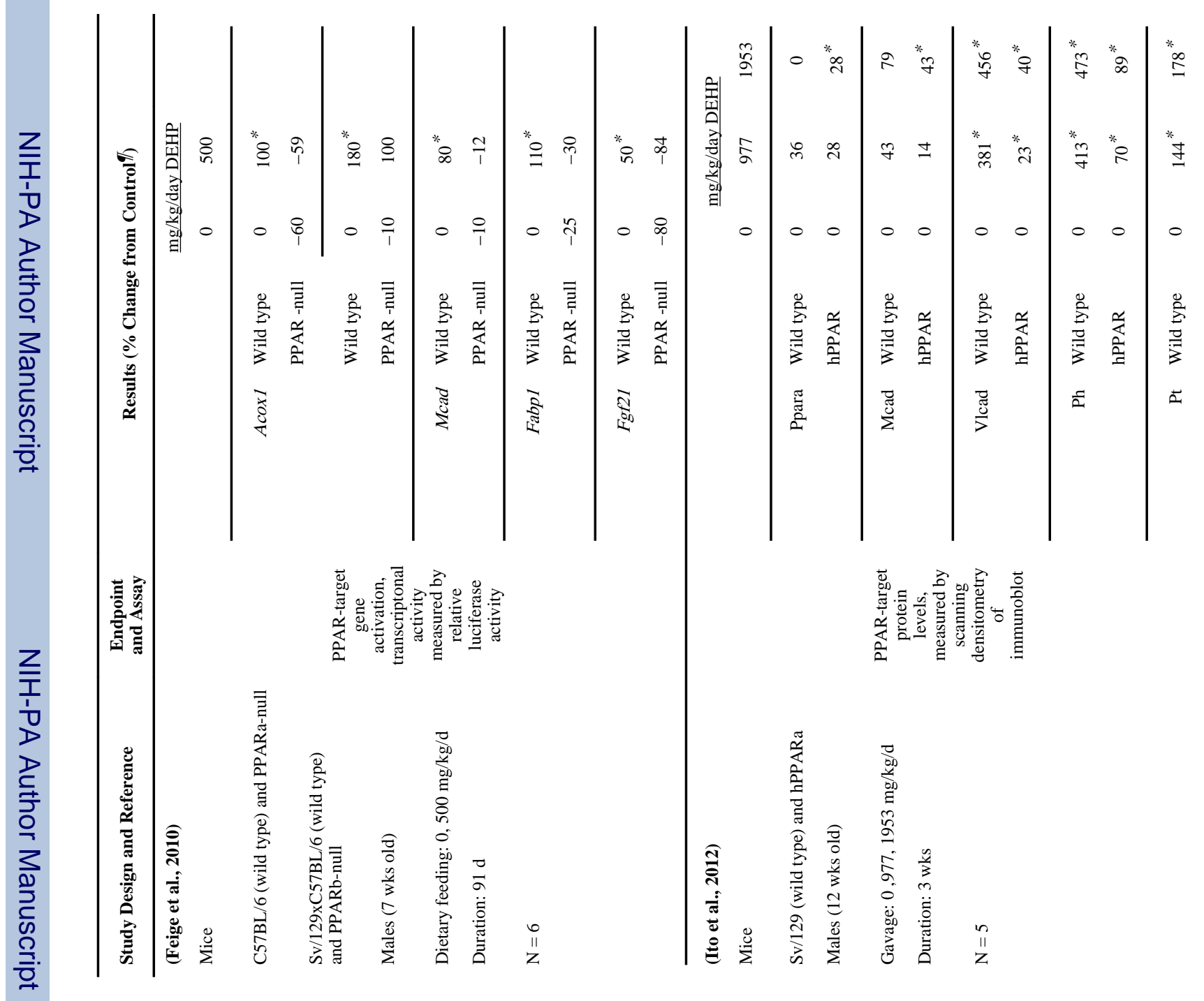




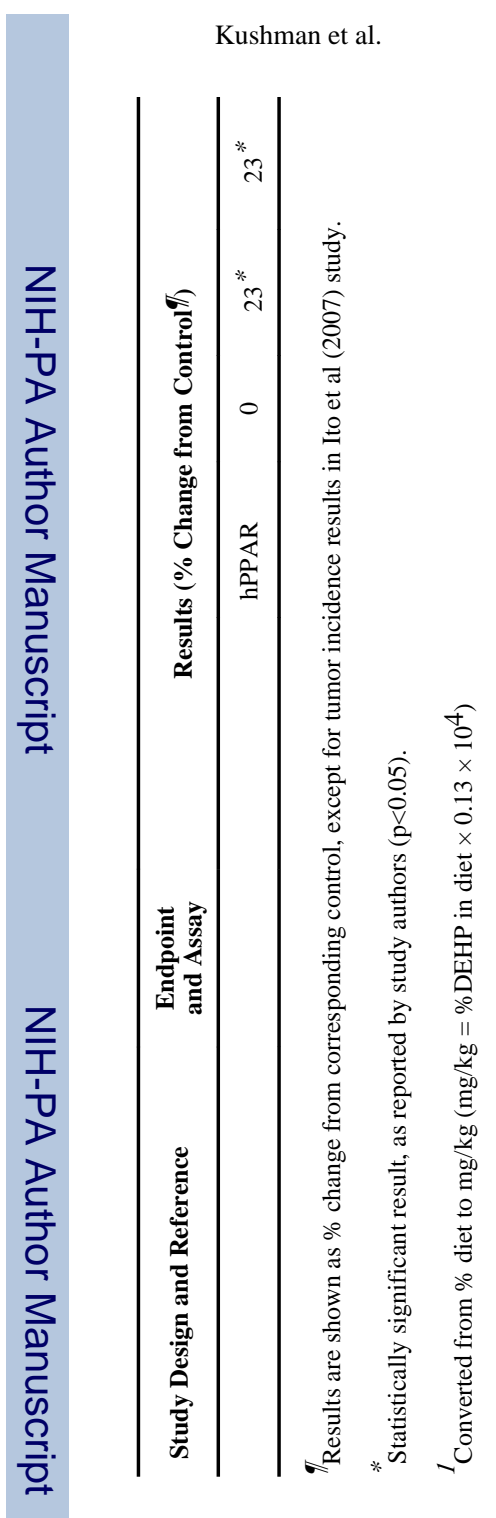

Page 25

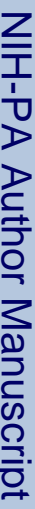

Regul Toxicol Pharmacol. Author manuscript; available in PMC 2014 November 01. 


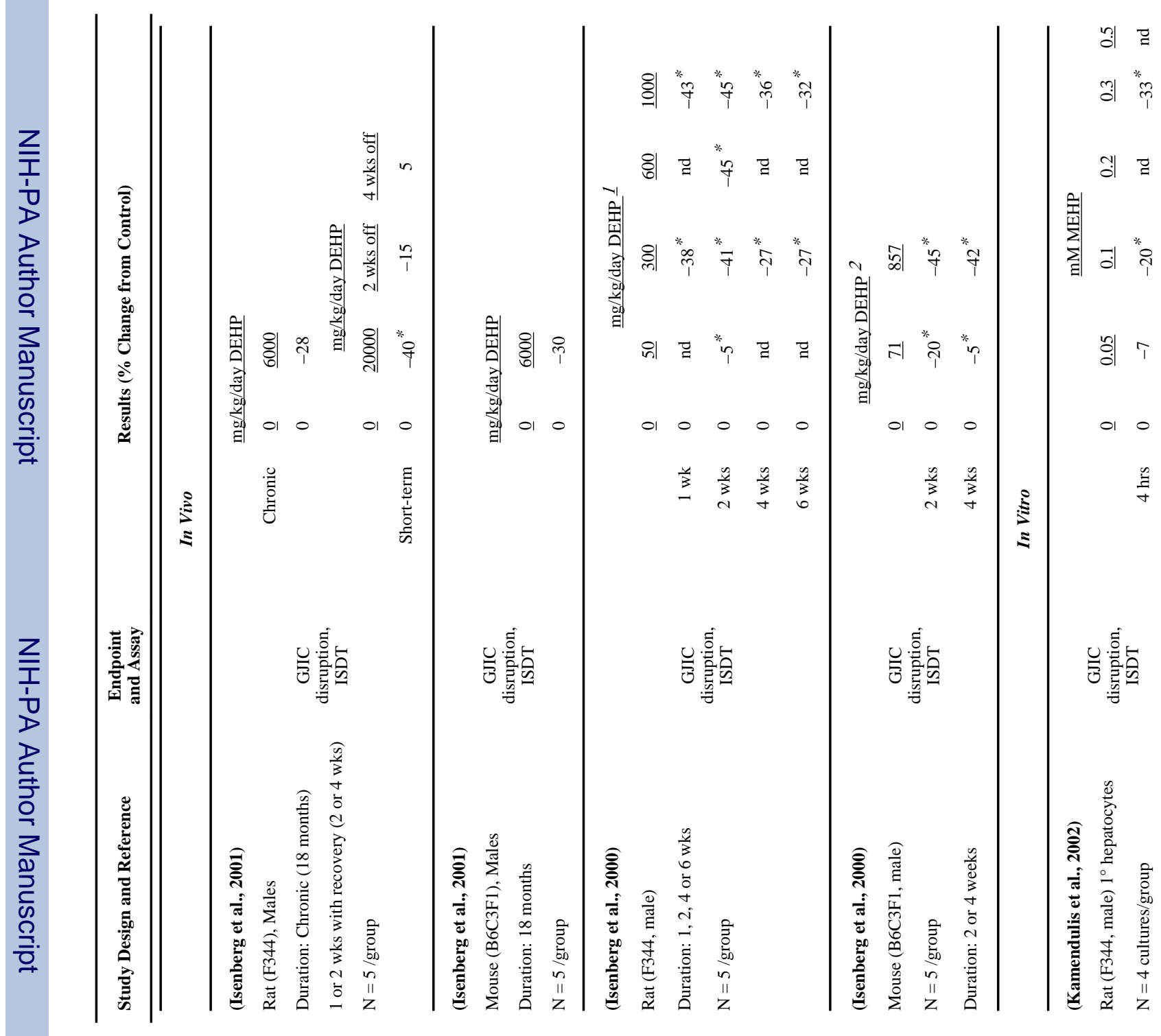

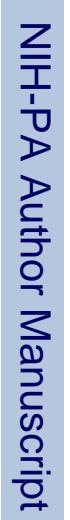




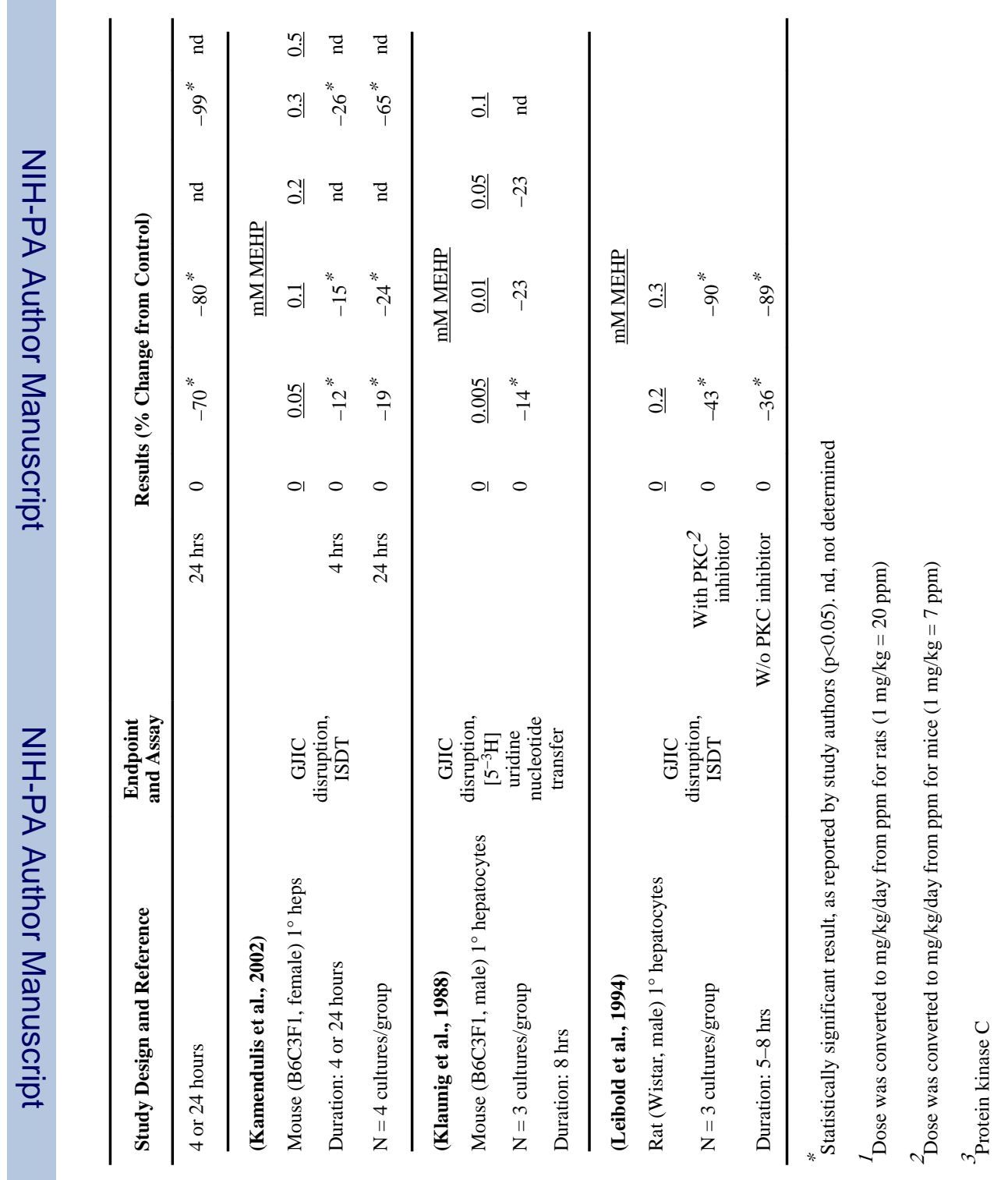

